\title{
Gubernamentalidad y poder pastoral
}

\section{Governmentality and Pastoral Power}

\author{
Mauro Benente ${ }^{1}$ \\ Universidad de Buenos Aires/ Universidad Nacional \\ de José C. Paz (Argentina)
}

Recibido: 06-02-16

Aprobado: 24-01-17

\section{Resumen}

En sus trabajos de fines de la década de 1970 Michel Foucault abordó la noción de gubernamentalidad para dar cuenta del funcionamiento del gobierno de los hombres. En su genealogía de estas tecnologías de gobierno encuentra un antecedente remoto en el poder pastoral y afirma que el pastorado es ajeno al pensamiento político de la Grecia clásica. En este trabajo desarrollo algunos problemas en la presentación que realiza de la obra de Platón. Foucault estudia la aparición del poder pastoral en las obras políticas de Platón, en particular en Político, y afirma que allí se rechaza al pastor como clave para explicar la actividad de gobierno, pero creo que sus argumentos no alcanzan para realizar esta afirmación.

Palabras-clave: Gubernamentalidad, Poder pastoral, Político, Tejedor.

\begin{abstract}
In his works of the late 1970s Michel Foucault used the notion of governmentality to explain the operation of the government of men. In his genealogy of these technologies government he finds a remote antecedent in the pastoral power, and he claims that the pastorate is foreign to the political thought of classical Greece. In this paper I develop some problems in the presentation made by Foucault of the work of Plato. Foucault studies the emergence of pastoral power in the political works of Plato, particularly in Statesman, and he claims that Plato rejects the pastor as a key to explain the activity of government, but I think that his arguments are not enough to make this assertion.
\end{abstract}

Key-words: Governmentality, Pastoral Power, Statesman, Weaver.

\footnotetext{
${ }^{1}$ (maurobenente@yahoo.com ) Doctor en Derecho por la Universidad de Buenos Aires. Becario Posdoctoral del CONICET. Docente de Teoría del Estado en la Facultad de Derecho de la Universidad de Buenos Aires y Profesor Titular de Filosofía del Derecho en la Universidad Nacional de José C. Paz. 


\section{Introducción}

Las temáticas del saber y la verdad, el poder y el gobierno, y la subjetivación, atraviesan de modo oblicuo toda la producción teórica de Foucault. Si bien estas variables aparecen interrelacionadas podría sostenerse que en los trabajos de la década de 1970, dedicados a las disciplinas, la biopolítica y la gubernamentalidad, hay una mayor intensidad en el análisis del poder y el gobierno. En este marco, a primera vista llama la atención que prácticamente no tematice sobre los autores más importantes de la teoría política antigua, moderna y contemporánea. Sin embargo, en varias ocasiones subrayó que su preocupación se situaba en el funcionamiento de las relaciones de poder y gobierno, para lo cual había que recurrir más a los archivos de instituciones, los expedientes y reglamentos, que a los pesos pesados de la teoría política. Sin embargo, en el marco de sus estudios sobre la gubernamentalidad Foucault se detuvo en la obra de Platón, en particular en la metáfora del pastor.

En el presente trabajo desarrollaré brevemente la noción de gubernamentalidad y también presentaré los contornos del poder pastoral, un antecedente del gobierno de los hombres que comienza a desplegarse con potencia en la modernidad. La hipótesis enunciada por Foucault indica que el pastorado como tecnología de gobierno fue ajena al pensamiento griego, marco en el cual sostiene que el propio Platón ha rechazado al pastor como grilla de inteligibilidad del gobierno. Además de reseñar esta hipótesis, mostraré algunas insuficiencias que encuentro en la presentación que hace Foucault del pastor al interior de la obra de Platón.

\section{El gobierno de los hombres}

Los estudios sobre la gubernamentalidad representan una discontinuidad con los análisis que se encontraban en trabajos anteriores, puesto las relaciones de poder ya no son analizadas bajo el paradigma de la relación de fuerzas, sino bajo el del gobierno de los hombres ${ }^{2}$. Tomando una fotografía de los textos se advierte que Foucault deja de referirse a las relaciones de poder y alude al gobierno, pero así como no había desplegado una teoría del poder, tampoco desarrolla una teoría del gobierno ${ }^{3}$. A partir de estos trabajos Foucault comenzó

2 Santiago Castro-Gómez, Historia de la gubernamentalidad. Razón de Estado, liberalismo y neoliberalismo en Michel Foucault, Bogotá, Siglo del Hombre Editores, 2010, pp. 17-27. David Alesio, La biopolítica foucaulteana: desde el discurso de la guerra hacia la grilla de la gubernamentalidad, "A Parte Rei. Revista de Filosofía, 60 (2008), p. 4.

${ }^{3}$ El gobierno alude a cierta perspectiva que hace inteligible los "intentos de los diferentes tipos de autoridades de influir en las acciones de los otros, en relación con objetivos de prosperidad nacional, armonía, virtud, productividad, orden social, disciplina, emancipación, autorrealización y así sucesivamente". Nikolas Rose, Identity, Genealogy, History [en Stuart Hall y Paul Du Guy, eds.: Questions of Cultural Identity, London, Sage Publications, 1996], pp. 134-135. 
a centrarse "no tanto en las relaciones de fuerzas, sino en las articulaciones que se dan entre tres dimensiones irreductibles unas a otras: el poder, el saber, la subjetividad"4. La constitución de los saberes y las subjetividades "ya no son vistos como meros epifenómenos del poder"s sino que se subraya la interrelación entre los tres dominios: "un gobierno sobre los sujetos, con la ayuda de saberes". Incluso, en Del gobierno de los vivos postuló que así como había desplazado el concepto de ideología por el de saber-poder ahora le interesaba moverse desde "la noción de saber-poder a la noción de gobierno a través de [par] la verdad"7. Además, a partir de estos años, comenzó a utilizar la noción de gobierno para referirse a la conducción de conducta de los otros, y cuando en los años ochenta aludió al poder lo hizo en esos términos ${ }^{8}$.

La noción de gubernamentalidad no es trabajada en los libros de Foucault y fue acuñada por primera vez en la clase del 1 de febrero de 1978 del curso Seguridad, territorio, población. Aunque el curso recién fue publicado en octubre del 2004, la clase fue traducida por Pasquale Pasquino -quien había asistido al curso- e incluida en el número 167-168 de la revista italiana Aut-Aut de septiembre-diciembre de 1978, año en el que Foucault publicó el resumen del curso donde tematizó brevemente sobre el concepto. La traducción al inglés de la clase apareció en el número VI del periódico Ideology and Consciousness en el otoño europeo de 1979 y a partir de la publicación de la clase y otros trabajos que la rodearon, aunque el curso todavía se encontraba inédito durante las décadas de 1980 y 1990 comenzaron a proliferar trabajos que emplearon y (re)conceptualizaron la noción de gubernamentalidad, muchos de ellos escritos por autores anglosajones nucleados en la red "Historia del presente," fundada en Londres en $1989^{9}$.

Foucault entiende por gubernamentalidad un conjunto de instituciones, procedimientos, análisis, reflexiones y tácticas, que configuran una modalidad de poder que tiene como blanco la población, como saber la economía política

${ }^{4}$ Santiago Castro-Gómez, Historia de la gubernamentalidad, Op. cit., p. 26.

5 Ídem.

${ }^{6}$ Frédéric Gros, Michel Foucault, Paris, Presses Universitaires de France, 1996, p. 84.

${ }^{7}$ Michel Foucault, Du gouvernement des vivants. Cours au Collège de France (1979-1980), Paris, Gallimard- Seuil, 2012, p. 12.

${ }^{8}$ En El sujeto y el poder sostuvo que el poder es una "estructura total de acciones dispuestas para producir acciones posibles: incita, induce, seduce, torna más simple o más dificultoso [...] una forma de actuar sobre la acción de un sujeto o acciones de los sujetos, en virtud de su propia acción o de ser capaces de acción. Un conjunto de acciones sobre otras acciones." Michel Foucault, The Subject and Power [en Hubert Dreyfus, y Paul Rabinow, Michel Foucault. Beyond structuralism and hermeneutics, Chicago, Chicago University Press, 1983], p. 220.

${ }^{9}$ Una buena reseña de la consolidación de estos estudios en Nikolas Rose, Pat O’Malley, Mariana Valverde, Governmentality, “Annual Review of Law and Social Science”, 2 (2006), pp. 92-97. Sobre la recepción de la obra de Foucault en Gran Bretaña, puede consultarse Colin Gordon, Foucault in Britain [en Andrew Barry, Thomas Osborne, Nikolas Rose, eds.: Foucault and political reason. Liberalism, neo-liberalism and rationalities of government, Chicago, Chicago University Press, 1996].

Araucaria. Revista Iberoamericana de Filosofía, Política y Humanidades, año 19, nº 37. Primer semestre de 2017. Pp. 45-64. ISSN 1575-6823 e-ISSN 2340-2199 doi: 10.12795/araucaria.2017.i37.03 
y como técnica específica los dispositivos de seguridad. También alude a la tendencia por la cual el gobierno comenzó a tener preeminencia por sobre la soberanía y las disciplinas. Finalmente refiere al proceso por el cual el Estado "se encontró poco a poco "gubernamentalizado»" gubernamentalidad Foucault aborda no solamente las prácticas de gobierno, sino su racionalidad. La gubernamentalidad alude a un tipo de ejercicio de poder guiado por una racionalidad y una práctica reflexiva, ${ }^{11}$ y por ello el foco de los estudios se encuentra "en el conocimiento ideal de los planes de gobierno, más que en los detalles acerca de cómo se implementan y cuáles son los efectos que podrían tener empíricamente" ${ }^{12}$. La racionalidad gubernamental es definida en un trabajo muy influyente de Gordon como "un modo o sistema de pensamiento sobre la naturaleza de la práctica de gobierno (quién puede gobernar; qué es gobernar; qué o quién es gobernado), capaz de hacer, de algún modo, esta actividad pensable y practicable, tanto por sus operadores como por sobre quienes es practicada"13. Lo que subyace a estas indagaciones es que "es posible analizar la racionalidad política, así como se puede analizar cualquier racionalidad científica" ${ }^{14}$.

En su estudio sobre el gobierno Foucault consulta las definiciones de diccionarios franceses de los siglos XIII a XV y encuentra que gobernar alude a hacer seguir una ruta, proporcionar víveres, conducir a otros, etc. Las conceptualizaciones son muy variadas, pero "no se gobierna jamás un Estado, no se gobierna jamás un territorio, no se gobierna jamás una estructura política. A los que se gobierna, de todas formas, es a las gentes, a los hombres, a los individuos o colectividades [...] A los que se gobierna son a los hombres"15. Además Foucault encuentra la emergencia de las artes de gobierno de los hombres en textos críticos de El Príncipe de Maquiavelo del siglo XVI, en los cuales se remarca que no solamente hay que gobernar el Estado, sino también la

${ }^{10}$ Michel Foucault, Sécurité, territoire, population. Cours au Collège de France (1977-1978), Paris, Gallimard- Seuil, 2004, pp. 111-112. Es así que "no es la «étatisation de la société» [estatización de la sociedad], sino más bien, y por el contrario, la «gouvernamentalisation de l'état» [gubernamentalización del Estado] lo que imprime la marca distintiva al presente al cual pertenecemos." Sandro Chignola, Foucault y la política de los gobernados. Gubernamentalidad, formas de vida, subjetivación, "Deus Mortalis", 9 (2010), p. 234.

${ }^{11}$ Cfr. Thomas Lemke, Foucault, Governmentality, and Critique, "Rethinking Marxism: A Journal of Economics, Culture \& Society,” 14, 3 (2002), p. 53; Barry Hindess, Discourses of power: From Hobbes to Foucault, Oxford, Blackwell, 1996, p. 106; Nikolas Rose, Powers of Freedom. Reframing political thought, Cambridge, Cambridge University Press, 1999, p. 7.

12 Pat O'Malley, Experimentos en gobierno. Analíticas gubernamentales y conocimiento estratégico del riesgo, "Revista argentina de sociología", 8 (2007), p. 155.

${ }^{13}$ Collin Gordon, Governmental Rationality: An Introduction [en Graham Burchell, Collin Gordon, Peter Miller, Eds.: The Foucault Effect. Studies in Governmentality, Chicago, Chicago University Press, 1991], p. 4.

${ }_{14}$ Michel Foucault, La technologie politique des individus [en Dits et écrits II, Paris, Gallimard, 2001, n³64], p. 1646.

${ }_{15}$ Michel Foucault, Sécurité, territoire, population, Op. cit., p. 126.

Araucaria. Revista Iberoamericana de Filosofía, Política y Humanidades, año 19, n 37. Primer semestre de 2017. Pp. 45-64. ISSN 1575-6823 e-ISSN 2340-2199 doi: 10.12795/araucaria.2017.i37.03 
casa, los conventos, a los niños. Hay una "pluralidad de formas de gobierno"16 y en consonancia con esto "muchas gentes gobiernan: el padre de familia, el superior de un convento, el pedagogo, el maestro sobre el niño o el discípulo: hay pues muchos gobiernos en relación a los cuales el del Príncipe gobernando su Estado no es más que una de sus modalidades" ${ }^{17}$. Este gobierno de los hombres, antes de desplegarse bajo el paradigma de la gubernamentalidad -en particular Foucault estudia las modalidades liberales y neoliberales-, presenta tres antecedentes: a- el poder pastoral; b- la técnica diplomático militar posterior a la Paz de Westfalia, c- las técnicas de policía en los siglos XVII y XVIII.

\section{El poder pastoral}

En Seguridad, territorio, población Foucault abordó por primera vez y con más detalle el poder pastoral, y también de 1978 datan dos conferencias dictadas en Tokio, "La filosofía analítica de la política" y "Sexualidad y soledad", en las cuales hay un importante tratamiento. En la conferencia Omnes et singulatim de 1979, y en El sujeto y el poder de 1982, hay breves desarrollos del pastorado, y aunque es un asunto sobre el que no volvió en tantas oportunidades mantuvo la categoría bajo análisis hasta 1984, el año de su muerte ${ }^{18}$.

Si bien la tradición rastrea en Grecia y Roma los parámetros para conceptualizar la política moderna y contemporánea, Foucault muestra que el primer registro del gobierno de los hombres, el pastorado, en términos generales no se ha desplegado en aquellas latitudes ${ }^{19}$. Es posible encontrar la emergencia del poder pastoral en el Oriente Mediterráneo a principios del 2000 a.c., pero fueron los hebreos quienes intensificaron la tecnología pastoral. A partir de estudios sobre el judaísmo, Foucault reconstruye estas características del pastorado hebreo: a- a diferencia de la divinidad griega, el poder del pastor

${ }^{16}$ Michel Foucault, Sécurité, territoire, population, Op. cit., p. 96.

17 Ídem.

18 Aludió por última vez al poder pastoral en "À propos de la généalogie de l'éthique: un aperçu du travail en cours", la versión francesa -que tiene modificaciones introducidas por Foucault- de "On the genealogy of Ethics: An overview of work in progress," entrevista que le realizaron Dreyfus y Rabinow en 1982. Allí indicaba el modo en que el cuidado de sí había perdido su carácter de práctica autónoma cuando fue capturada por las prácticas del poder pastoral. Cfr. Michel Foucault, À propos de la généalogie de 1'éthique: un aperçu du travail en cours [en Dits et écrits II, Paris, Gallimard, 2001, n 344], p. 1448. En el mismo sentido, Michel Foucault, Usage des plaisirs et techniques de soi [en Dits et écrits II, Paris, Gallimard, 2001, n 338], pp. 1363-1364; Histoire de la sexualité 2. L'usage des plaisirs, Paris, Gallimard, 1984, pp. 16-17.

${ }_{19}$ En Omnes et singulatim postula que la idea que "el rey o el jefe es un pastor seguido de un rebaño de ovejas no era familiar en los griegos y en los romanos [...] Groseramente hablando, podemos decir que la metáfora del rebaño está ausente en los grandes textos políticos griegos o romanos." Michel Foucault, «Omnes et singulatim»: vers une critique de la raison politique [en Dits et écrits II, Paris, Gallimard, 2001, n 291] p. 955. 
no se ejerce sobre el territorio sino sobre una multiplicidad en movimiento ${ }^{20}$; b- el pastor tiene la función de reunir a individuos dispersos, para luego guiar al rebaño ${ }^{21}$; c- el pastor provee todo lo necesario para la subsistencia del rebaño. Es un poder de cuidado, que vela por cada uno en vistas de su salvación ${ }^{22}$; d- el poder pastoral es individualizante porque vela por el conjunto del rebaño, pero también por cada uno de los animales. Esto configura la paradoja del pastor: "debe tener los ojos sobre todos y sobre cada uno, omnes et singulatim, que será precisamente el gran problema de las técnicas de poder en el pastorado cristiano y de las técnicas de poder, digamos, modernas, tal como ellas están planificadas en las tecnologías de la población"23. Este poder individualizante busca la obediencia de cada individuo pero también "conocerlo, descubrirlo, hacer aparecer su subjetividad y estructurar la relación que él tiene con él mismo y con su propia conciencia" 24 .

El pastorado comienza a desplegarse con potencia cuando una comunidad religiosa se constituye como una Iglesia, esto es, "como una institución con pretensiones de gobierno de los hombres en su vida cotidiana so pretexto de dirigirlos a la vida eterna en el otro mundo, y esto a escala [...] de la humanidad en su conjunto"25. La Iglesia Católica desarrolló un dispositivo de poder que no tuvo competición entre los siglos III y XVIII, y tuvo continuidades con el pastorado hebreo, puesto que en ambos casos, el pastor debe velar por la salvación de todo el rebaño y de cada una de las ovejas. Sin embargo también incluyó novedades: a) el cristianismo establece una particular relación con la ley, puesto que mientras el ciudadano griego se dejaba dirigir por la ley pero su relación no era de obediencia sino de respeto, el pastorado organizó la "instancia de la obediencia pura, la obediencia como tipo de conducta unitaria, conducta altamente valorada y que tiene lo esencial de su razón de ser en sí misma"26. El cristianismo no es una religión de ley sino de voluntad divina y "la relación de la oveja con el que la dirige es una relación de dependencia integral"27. La obediencia es la renuncia de la propia voluntad e implica un mérito: "permanecer obediente es la condición fundamental de todas las otras virtudes"28; b) respecto de la relación con la verdad, el pastorado cristiano

${ }^{20}$ Michel Foucault, Sécurité, territoire, population, Op. cit., pp. 129-130; «Omnes et singulatim»: vers une critique de la raison politique, Op. cit., pp. 956-957; Sexualité et pouvoir [en Dits et écrits II, Paris, Gallimard, 2001, nº 233], p. 561.

${ }^{21}$ Michel Foucault, «Omnes et singulatim»: vers une critique de la raison politique, Op. cit., p. 957.

22 Michel Foucault, Sécurité, territoire, population, Op. cit., pp. 130-132; Sexualité et pouvoir, Op. cit., p. 561.

${ }^{23}$ Ibidem, p. 132.

${ }^{24}$ Michel Foucault, La philosophie analytique de la politique [en Dits et écrits II, Paris, Gallimard, 2001, no 232], p. 549.

25 Michel Foucault, Sécurité, territoire, population, Op. cit., pp. 151-152.

${ }^{26}$ Ibidem, p. 177.

27 Ibidem, p.178.

${ }^{28}$ Michel Foucault, Sexualité et pouvoir, Op. cit., pp. 563-564. 
comparte con los griegos la enseñanza a partir del ejemplo, y bajo una lógica individual. Sin embargo, hay dos elementos que son bien distintivos de la enseñanza del pastorado: 1- debe ser una dirección de la vida cotidiana, que debe transitar "por una observación, una vigilancia, una dirección ejercida a cada instante y de la manera menos discontinua posible"29; 2- el pastor, además de enseñar la verdad, debe encargarse de dirigir la conciencia, remarcando así los lazos de dependencia ${ }^{30}$.

Podrían desarrollarse con más precisión los contornos del poder pastoral, este primer antecedente del gobierno de los hombres, pero me interesa concentrarme en el modo en que Foucault descarta el paradigma del pastorado en la reflexión política de la Gracia antigua y clásica. En particular, me interesa enfatizar algunas deficiencias en los desarrollos de Foucault al momento de negar la presencia del pastorado en el pensamiento político griego.

\section{3.a. El pastorado en el pensamiento griego}

De acuerdo con la lectura que propone Foucault, la metáfora del pastorrebaño no ha sido en Grecia un modelo apropiado ni utilizado para explicitar la especificidad del gobierno. Sin embargo, es posible dar cuenta de tres grandes grupos de excepciones: a- el vocabulario homérico; b- la tradición pitagórica; c- los fragmentos de Arquitas de Anfis editados por Otto Friedrich Gruppe y algunos diálogos de Platón.

En el vocabulario homérico la metáfora del pastor aparece en numerosas oportunidades. En la Ilíada la metáfora se utiliza para aludir a reyes y jefes militares -en especial a Agamenón- bajo el rótulo de "pastor de huestes" "31. En la Odisea, y también en varias oportunidades para caracterizar a Agamenón, la metáfora que más se emplea es la de "pastor de los pueblos" o "pastor de las gentes" aunque también se alude al "pastor de los soldados"32. Sin embargo Foucault le quita importancia a estas menciones porque sostiene que se trata de un apelativo ritual que se encuentra en gran parte de la literatura indoeuropea ${ }^{33}$. Por su lado, en la tradición pitagórica también encontramos referencias al pastor y se subraya que de acuerdo con su etimología, ley (nomos) proviene de pastor

\footnotetext{
${ }^{29}$ Michel Foucault, Sécurité, territoire, population, Op. cit., p. 184.

30 Ibidem, pp. 184-185.

${ }^{31}$ Homero, Ilíada, Madrid, Gredos, 1996, Canto I 263, Canto II 85, 105, 243, 254, 772, Canto IV, 296, 413, 455, Canto V, 144, 513, 566, 570, Canto VI, 214, Canto VII, 230, 469, Canto IX, 81, Canto X, 3, 73, 406, Canto XI, 92, 187, 202, 506, 578, 598, 651, 697, 842, Canto XIII, 411, 600, XIV, 423, 516, Canto XV 262, XVI, 2, XVII, 348, XIX, 35, 251, 386, Canto XX, 110, Canto XXII, 277, Canto XXIII, 389, 411, XXIV, 654

${ }^{32}$ Homero, Odisea, Madrid, Gredos, 1993, Canto III, 156, 469, Canto IV, 24, 528, 532, Canto XIV, 497, Canto XV, 151, Canto XVII, 109, Canto XVIII, 70, Canto XX, 107, Canto XXIV, 456.

${ }^{33}$ Michel Foucault, Sécurité, territoire, population, Op. cit., p. 140.
} 
(nomeus), a la vez que se enuncia que el pastor distribuye el alimento y dirige al rebaño, tarea similar a la desplegada por el magistrado. Aquí el argumento de Foucault para descartar la centralidad del pastor en el pensamiento griego es que la "tradición pitagórica es una tradición si no marginal, al menos limítrofe"34. Esto tal vez sea así, pero el problema es que Foucault no brinda argumentos para sustentar el supuesto carácter periférico, algo que se agrava si tenemos en cuenta que Aristóteles, en el primer libro de Metafísica, indica que dentro de las influencias de Platón se encuentran Cratilo, Heráclito y Sócrates, pero también los desarrollos pitagóricos ${ }^{35}$.

Si bien creo que el argumento para deshacerse de las referencias pitagóricas es insuficiente, Foucault tiene por delante la difícil tarea de descartar los fragmentos de Arquitas y fundamentalmente los diálogos de Platón, serie a la que nomina como "vocabulario político clásico". En los fragmentos de Arquitas la metáfora está presente pero por influencia hebrea -lo que confirma la tesis de la procedencia oriental de la metáfora-, y el pastor no aparece en Demóstenes, Isócrates, ni en Aristóteles, por lo que en "el vocabulario político clásico de Grecia, la metáfora del pastor es una metáfora rara" "36. No obstante el propio Foucault reconoce una notable excepción: la obra de Platón. Sin embargo, antes de avanzar sobre este punto, llama la atención que no repare en la aparición de la metáfora en Ciropedia de Jenofonte. En el libro primero, el discípulo de Sócrates parece distanciarse del paralelismo entre el gobernante y el pastor, puesto que afirma que el rebaño es más obediente que los hombres y no hay ejemplos históricos de rebelión de los rebaños como sí se encuentran entre los hombres ${ }^{37}$. Sin embargo, apropiándose de la metáfora en el libro VIII parafrasea a Ciro y se hace eco del paralelismo entre el rey y el pastor: "se recuerda su dicho de que eran similares los oficios del buen pastor y del buen rey. Pues afirmaba que el pastor debía sacar provecho de su ganado asegurando su felicidad -la felicidad propia del ganado-, y que, de la misma manera, el rey debía sacar provecho de ciudades y hombres asegurando su felicidad" 38 .

\footnotetext{
${ }^{34}$ Ibidem, p. 164.

35 Aristóteles, Metafísica, Madrid, Gredos, 1970, 987 a-b.

${ }^{36}$ Michel Foucault, Sécurité, territoire, population, Op. cit., p. 142
}

${ }_{37}$ Así se lee que "seguíamos reflexionando sobre el hecho de que gobernantes son los boyeros de sus bueyes, los yegüeros de sus caballos y que todos los que reciben el nombre de pastores podrían también ser considerados razonablemente gobernantes de los animales a cuyo cuidado están; pues bien, nos parecía apreciar que todos estos rebaños obedecen de mejor grado a sus pastores que los hombres a sus gobernantes. En efecto, los rebaños van exclusivamente por donde los pastores los dirigen, pacen en los lugares a los que los conducen y se mantienen alejados de aquellos de los que los apartan. Además, permiten a los pastores hacer el uso que quieran de los productos que se obtienen de ellos y aún no tenemos noticias de que nunca un rebaño se rebelara contra su pastor, ni para desobedecerle ni para impedirle hacer uso de sus productos, sino que, al contrario, los rebaños son más ariscos con cualquier extraño que con quienes los gobiernan y sacan provecho de ellos. Los hombres, en cambio, contra nadie se levantan más que contra aquellos en quienes noten intención de gobernarlos.” Jenofonte, Ciropedia, Madrid, Gredos, 1987, I 1, 2.

${ }^{38}$ Jenofonte, Ciropedia, Op. cit., VIII 2, 14. 
De modo más impreciso la metáfora también se enuncia en algunos pasajes de Memorabilia, tanto parafraseando a Sócrates ${ }^{39}$ cuanto en la presentación de Agamenón como pastor de los pueblos en los poemas de Homero ${ }^{40}$.

Teniendo en cuenta este breve desarrollo me parece que Foucault descarta de modo apresurado la influencia del pensamiento de Pitágoras, y resulta llamativa la omisión de Jenofonte. Quizás estas débiles argumentaciones y omisiones deberían matizar la hipótesis sobre la inexistencia del pastorado en el pensamiento griego, pero creo que todavía más problemático es el tratamiento que realiza de la obra de Platón.

\section{3.b. El pastor en la obra de Platón.}

En sus estudios sobre el pastorado Foucault abordó la obra de Platón, pero también la analizó con anterioridad y especialmente con posterioridad. Con anterioridad, de modo disperso y sin mayor profundidad, insistió en varias oportunidades en que ha sido Platón el primero en separar al saber del poder, mito que solamente a partir de la filosofía de Nietzsche comenzó a desmontarse ${ }^{41}$. Con posterioridad, de manera más sistemática y detallada, la abordó en sus estudios sobre el cuidado de sí-analizando especialmente Alcibíades I, un diálogo de dudosa autoría- ${ }^{42}$, la parrhesía política -abordando varios diálogos y la dudosa carta VII- ${ }^{43}$ y la parrhesía ética -trabajando especialmente con Alcibiades I y Laques- ${ }^{44}$.

Sobre la temática que aquí nos ocupa, hay varios diálogos en los cuales el magistrado es asimilado al pastor: Critias, República, Político y Leyes. Si tenemos en cuenta esta lista podría sostenerse que a excepción de Sofista -donde hay reflexiones sobre la política hacia el final de la conversación-, Platón utiliza la metáfora del pastor en todos sus diálogos políticos, lo que hace pensar que la excepción que plantea Foucault se parece mucho a una regla. De todos modos, dejando por el momento en suspenso el tratamiento de Político, destaca que fue empleada en tres sentidos:

${ }^{39}$ Jenofonte. Recuerdos de Sócrates. Memorabilia, Madrid, Gredos, 1993, I 2, 32.

${ }^{40}$ Ibidem, III 2, 1.

${ }^{41}$ Michel Foucault, L'ordre du discours, Paris, Gallimard, 1971, pp. 17-18; De l'archéologie à la dynastique [en Dits et écrits I, Paris, Gallimard, 2001, nº 119], p. 1282; La vérité et les formes juridiques [en Dits et écrits I, Paris, Gallimard, 2001, $\mathrm{n}^{\circ}$ 139], p. 1458; Les confessions de Michel Foucault (Entretien avec R.P. Droit) [en Le Point, 01/07/2004. Disponible en http://www.lepoint.fr/ actualites-chroniques/2004-07-01/les-confessions-de-michel-foucault/989/0/116571]

${ }^{42}$ Michel Foucault, Subjectivité et vérité. Cours au Collège de France (1980-1981), Paris, Gallimard- Seuil, 2014; L'herméneutique du sujet. Cours au Collège de France (1981-1982), Paris, Gallimard- Seuil, 2001.

${ }^{43}$ Michel Foucault, Le gouvernement de soi et des autres. Cours au Collège de France (19821983), Paris, Gallimard-Seuil, 2008.

${ }^{44}$ Michel Foucault, Le courage de la vérité. Cours au Collège de France (1983-1984), Paris, Gallimard-Seuil, 2009. 
a- La metáfora del pastor aparece en Critias, un diálogo inconcluso que tematiza sobre la guerra entre Atenas y Atlántida - un imaginario imperio occidental ubicado más allá de las columnas de Heracles-. Al parecer es un diálogo de vejez, escrito con inmediata posterioridad al Timeo y seguido por Filebo y Leyes, o directamente por este último. Aquí el pastorado aparece superficialmente cuando Platón designa una modalidad bienaventurada de poder ejercida por los Dioses durante los primeros días de existencia de los hombres. Es así que pone en boca de Critias: "en una ocasión, los dioses distribuyeron entre sí las regiones de toda la tierra por medio de la suerte [...] Una vez que cada uno obtuvo lo que le agradaba a través de las suertes de la justicia, poblaron las regiones y, después de poblarlas, nos criaban como sus rebaños y animales, como los pastores hacen con el ganado, sólo que no violentaban cuerpos con cuerpos, como los pastores apacientan las manadas a golpes, sino como es más fácil de manejar un animal: dirigían desde la proa"45. Como se lee, la metáfora del pastor se aplica para dar cuenta de un gobierno divino sobre los hombres, que con aires de familia ya había sido enunciado en Político y que más adelante desarrollaré.

b- Otra aparición de la metáfora se lee en Leyes, el diálogo más largo de Platón -dos libros más extenso que República-, el último de sus diálogos políticos, el único que se desarrolla tras la muerte de Sócrates, y posiblemente inmediato anterior a Epinomis, que en general se considera su último diálogo ${ }^{46}$. En el libro $X$, que se inicia con un estudio sobre los delitos a la propiedad privada y de impiedad pero que desemboca en un análisis del fundamento de la legislación y el orden político, Platón le hace decir al Ateniense que el magistrado es tenido como un pastor, pero rápidamente aclara que no instituye la ciudad ni dicta las leyes más importantes ${ }^{47}$. De este modo, en la lectura que propone Foucault, el magistrado en tanto que pastor es un simple personaje secundario y subordinado.

c- En tercer lugar, se encuentran las referencias de República, que respetando el orden cronológico debería haber presentado en primer lugar. La reseña que hace Foucault de Critias y de Leyes no es errónea, y en esos diálogos efectivamente no se posiciona al pastorado como grilla de inteligibilidad de la actividad política. Sin embargo, la presentación de República no resulta tan precisa y por ello preferí dejarla para el final, a modo de preludio a los

45 Platón, Critias [en Diálogos VI, Madrid, Gredos, 1992], 109 b-c.

${ }^{46} \mathrm{La}$ datación del texto no es sencilla pero pueden reconstruirse algunas referencias: Aristóteles afirmó que es posterior a República, Diógenes de Laercio sostuvo que su edición fue póstuma, y pseudo Olimpiodoro agregó que se trataba de un borrador que Filipo de Opunte, uno de sus discípulos, organizó y transcribió. Sobre la autenticidad y ubicación temporal de la obra ver Juan Manuel Pabón, Manuel Fernández-Galiano, Introducción [en Platón, Leyes, Centro de Estudios Políticos y Constitucionales, Madrid, 1999], pp. VII-XVIII; Francisco Lisi, Introducción [en Platón, Diálogos VIII. Leyes, Madrid, Gredos, 1999], pp. 9-21.

${ }^{47}$ Platón, Leyes. Diálogos IX, Madrid, Gredos, 1999, 905e, 906 a-b-c. 
problemas de Político. Hay tres hipótesis sobre la edición de República, el primer diálogo estrictamente político de Platón: a- existieron dos ediciones, una aparecida alrededor del 390 a.c. que tenía el libro I íntegro y lo esencial de los libros II, III y IV, mientras que hacia el 370 a.c. apareció la edición que hoy conocemos; b- el primer libro se publicó en el 390 a.c. con el título de Trasímaco, y luego fue integrado a la edición del 370 a.c., c- la redacción llevó unos 20 años y recién en 370 a.c. apareció la obra completa, sin publicaciones parciales previas. El problema central del diálogo es la justicia, tanto en un plano individual cuanto colectivo, pero aquí me focalizaré en el pastorado como metáfora del gobierno.

En el libro I, Trasímaco de Calcedonia introduce la metáfora del pastor para sostener que era ingenuo creer que el pastor atendía a sus ovejas no por su propio interés sino por el bien de los animales ${ }^{48}$. Como reseña Strauss, efectivamente el pastor se preocupaba por las ovejas pero para que sean más jugosas y sabrosas para sus dueños - que no eran los pastores ${ }^{49}$ - Frente a esta argumentación Sócrates replicaba que la descripción no se ajustaba al verdadero pastor y era erróneo creer que "éste apacienta las ovejas en tanto pastor, sin mirar a lo que es mejor para las ovejas [...] el arte del pastor no cuida sin duda de ninguna otra cosa que de aquella con respecto a la cual está organizada, a fin de procurarle lo mejor" ${ }^{50}$. La frase puesta en la boca de Sócrates no termina aquí sino que se enuncia una poderosa analogía con el político: "del mismo modo estoy convencido de que es forzoso estar de acuerdo en que todo gobierno, en tanto gobierno, no atiende a ninguna otra cosa que al sumo bien de aquel que es su gobernado y está a su cuidado, trátese del gobierno del Estado o de ámbitos particulares" ${ }^{\text {"51 }}$. Frente a este pasaje, ¿qué hace Foucault para mantener que la metáfora del pastor es ajena al pensamiento griego? Lo adjudica, sin mayor desarrollo, al conocimiento que tenían Sócrates y Platón del pensamiento pitagórico ${ }^{52}$. Aquí hay un punto bastante oscuro, puesto que si el pensamiento pitagórico era tan marginal no se explica su influencia en un diálogo tan importante como República. Además, la metáfora es

${ }^{48}$ Le parecía muy torpe que Sócrates creyera "que los pastores y los boye ros atienden al bien de las ovejas y las vacas, y las engordan y cuidan mirando a otra cosa que al bien de los amos y al de ellos mismos; así como también estimas que los gobernantes de los Estados -los que gobiernan verdaderamente- piensan acerca de los gobernados de otro modo que lo que se ha establecido respecto de las ovejas y que los atienden día y noche he de otra manera que de aquella que les a provechará a ellos mismos" Platón, República. Diálogos IV, Madrid, Gredos, 1988, 343a-d.

49 Leo Strauss, The City and Man, Chicago, Chicago University Press, 1964, pp. 81-82. Del mismo modo, cuando Badiou trata este pasaje le hace decir a Trasímaco: "pareces creer que pastores y boyeros sólo tienen ojos para el bienestar de los ovinos y de los bovinos, que es sólo para complacer a las señoras ovejas y a los señores toros que los ceban y los cuidan. Es grotesco, mi pobre amigo. Lo hacen sólo para que su amo, el propietario de esas bonitas bestias cornudas y lanudas, saque de eso un enorme provecho." Alain, Badiou, La República de Platón, Buenos Aires, Fondo de Cultura Económica, 2013, p. 51.

${ }^{50}$ Platón, República, Op. cit., 345c-d.

51 Ibidem, 345d.

${ }^{52}$ Michel Foucault, Sécurité, territoire, population, Op. cit., p. 144. 
presentada por Trasímaco y no es rechazada sino confirmada por Sócrates, quien no declina de presentar al político como pastor sino que modifica sus objetivos en la tarea de crianza. De todos modos, continuando con las oscuridades en el abordaje del diálogo, el pastor aparece en otros pasajes no reseñados por Foucault, aunque sin implicancias políticas ${ }^{53}$. Sin embargo, en la última mención hay un uso que asimila los gobernantes a los pastores: Glaucón, sobre la función de los guardianes auxiliares acuerda con Sócrates y Adimanto que "en nuestro Estado los auxiliares sirvieran a los gobernantes, que son como los pastores del Estado" 54 . Ampliando los alcances de la comparación entre guardianes auxiliares -caracterizados como perros- y perfectos guardianes, Soares agrega que "éstos últimos constituirán la elite de los futuros gobernantes de la polis, comparables ya no con los perros sino con los pastores" ${ }^{25}$. En este pasaje del diálogo no se profundiza sobre la asimilación entre gobernantes y pastores pero la metáfora es clara, no es descartada ni rechazada, pero a pesar de ello Foucault no la menciona. De todos modos, los problemas en la restitución del pastor en la obra de Platón no se limitan a las omisiones de República.

\section{3.c. El pastor en Político}

Los personajes que participan de Político son los mismos que los de Sofista: Sócrates, el matemático Teodoro, el Extranjero de Elea y el Joven Sócrates, aunque los dos primeros intervienen solamente en el inicio de la conversación. También está presente Teeteto, pero no habla. Si bien se cree que el diálogo aparece de modo inmediatamente posterior a Sofista esto no aclara totalmente la fecha de publicación, que puede ubicarse sin mayores discusiones entre el segundo y el tercer viaje que realiza Platón a Siracusa, es decir entre el 366 y el 362 a.c. ${ }^{56}$ En términos generales podría sostenerse que si en República casi no se encuentran reflexiones conceptuales sobre la ley y la atención se centra en la formación de los perfectos guardianes, en Político se recapacita sobre la ley y su importancia en el entramado de la política ${ }^{57}$. La política se presenta como un arte o una ciencia de ejercer el gobierno, y el diálogo gira alrededor de su especificidad.

53 Platón, República, Op. cit., 359c-d, 370d, 399d, 416a.

${ }^{54}$ Ibidem, 440d.

${ }_{55}$ Lucas Soares, Platón y la política, Madrid, Tecnos, 2010, p. 121.

${ }_{56}$ María Isabel Santa Cruz, Introducción [en Platón, Diálogos V, Madrid, Gredos, 1988], p. 486.

57 Jean-François Balaudé, Le triptyque Republique, Politique, Lois: perspectives [en Jean-François Balaude, Ed., D'une cite possible. Sur le Lois de Plato, Nanterre, Presses de Nanterre, 1995], p. 33. Grube recuerda que con una educación que forme a los ciudadanos rectamente no serían necesarios los códigos legales y a lo largo de República "mantuvo Platón que la educación era más importante que la ley." George Grube, El pensamiento de Platón, Madrid, Gredos, 1994, p. 410. Colli agrega que la apuesta por la educación que se lee en República se explica por la aspiración a una polis ideal, algo que no aparece en Político y por ello "la moral cede su lugar al derecho." Giorgio Colli, Platón politico, Madrid, Siruela, 2008, p. 148. 
La primera conceptualización que se brinda sobre el arte de la política se explica a través del paralelismo con la actividad desarrollada por el pastor. Sin embargo, de acuerdo con la lectura que propone Foucault, cuando da cuenta de la especificidad del magistrado Platón embate de modo directo y frontal contra la metáfora del pastor. Muy hábilmente Foucault presenta el diálogo indicando que Platón no se pregunta si el pastorado puede caracterizar a tal o cual magistrado, sino si puede dar cuenta del magistrado por excelencia, si permite mostrar su especificidad. La pregunta es si lo distintivo del poder político puede explicarse con el modelo de la relación entre el pastor y el rebaño.

Se enuncia una aproximación preliminar del político como pastor de los hombres $^{58}$, y se alude a la política como la "ciencia de la crianza colectiva de hombres" "Sin embargo, el Extranjero advierte que con esta definición no se llega a aquello que distingue a la figura del magistrado, puesto que es una actividad compartida por otros integrantes de la polis como médicos, gimnastas, pedagogos y fundamentalmente sofistas ${ }^{60}$. Como subrayan Deleuze y Guattari "si se trata de hacerse cargo del bienestar de los hombres, hay muchos pretendientes que se presentan como el amigo del hombre, el campesino que lo alimenta, el tejedor que lo viste, el médico que lo cura, el guerrero que lo protege" ${ }^{\text {. }}$. Sin embargo la conceptualización del político como pastor es errónea no solamente porque otras artes también se ocupan del cuidado de los hombres, sino también porque "un pastor propiamente dicho se ocupa de todo, mientras que el político no se ocupa de todo"62. Finalmente, mientras el rebaño y el pastor son de diferente naturaleza, el pastor y sus gobernados son igualmente hombres por lo que si hubiera un pastor de los hombres debería ser de una naturaleza distinta, argumento que prepara el terreno para la presentación de pastor divino. En medio de la pregunta por la esencia de la actividad del magistrado ${ }^{63}$, nos encontramos con un relato mitológico que combina tres leyendas: a) el cambio en la dirección de los astros, motivado por un enojo de Zeus con Tiestes; b) la era de Cronos, que popularmente era asociada con una Edad de Oro y felicidad; c) el relato de los hijos de la tierra, que supone que los hombres brotaron y nacieron de la tierra ${ }^{64}$.

\footnotetext{
${ }_{58}$ Platón, Político [en Diálogos V, Madrid, Gredos, 1988], 257a-268d.

59 Ibídem, 267d.

${ }^{60}$ Lucas Soares, Platón y la política, Op. cit., p. 188.

${ }^{61}$ Gilles Deleuze, Félix Guattari, Qu' est-ce que la philosophie?, Paris, Les éditions de minuit, 1991, p. 15.

${ }^{62}$ Cornelius Castoriadis, Sobre el político de Platón, Madrid, Trotta, 2004, p. 44.

${ }^{63}$ El Extranjero postula que es necesario indagar cuál es el rasgo distintivo de los reyes y de los magistrados, puesto que de lo "que se trata es de saber si entre los demás pastores hay alguno que, poseyendo el nombre de otra arte, afirme frente a alguien, y así lo figure, que comparte en común con él la crianza del rebaño [...] Los comerciantes por ejemplo, los agricultores, los panaderos, todos ellos $\mathrm{y}$, además de ellos, los maestros de gimnasia y el género de los médicos, ¿te das cuenta de que todos, sin excepción, vendrían a disputar, y con justa razón, a enfrentarse enérgicamente a esos pastores de asuntos humanos a los que llamamos políticos, alegando que ellos mismos se preocupan por la crianza humana y, más aún, no sólo en lo que toca a los hombres que forman al rebaño, sino también a los gobernantes mismos?" Platón, Político, Op. cit, 267e-268a.

${ }^{64}$ Lucas Soares, Platón y la política, Op. cit., p. 189. A diferencia de la mayoría de las
} 
En un primer momento, en la era de Cronos, el mundo giraba sobre sí mismo, en el sentido de la felicidad, pero cuando empezó a girar en sentido inverso vinieron los tiempos difíciles ${ }^{65}$. En la edad de Cronos, dios apacentaba a los hombres cual pastores divinos y "cuando el dios los apacentaba no había regímenes políticos" ${ }^{\prime 6}$. Aquí la divinidad guarda una similitud con el pastor ${ }^{67}$, pero de acuerdo con Foucault en este pasaje de la historia no hay política, sino que la política emerge cuando se disuelve la era de Cronos: "los dioses se han retirado y los hombres están obligados a dirigirse los unos a los otros, es decir que tienen necesidad de política y de hombres políticos. Pero, y aquí el texto de Platón es muy claro, esos hombres que están ahora a cargo de otros hombres no están por encima del rebaño como los dioses podrían estar por encima de la humanidad. Ellos mismos forman parte de los hombres y no se los puede considerar como pastores"68. De este modo, Platón pone en boca del Extranjero que el mito ha servido para aclarar quién es digno de recibir el título del pastor, subrayando que "la figura del pastor divino es demasiado grande para parangonarla al rey y que nuestros políticos actuales son mucho más semejantes por su naturaleza a los hombres por ellos gobernados y que la cultura y la educación de la que tienen parte se aproximan mucho más a la de los gobernados" $"$.

Si no es posible asimilar la tarea del político a la del pastor, se la asemeja a la del tejedor: lo propio del político es unir, entrelazar, confeccionar una buena trama. Es así que en la última intervención del Extranjero, se lee que "el fin del tejido de la actividad política: [es] la combinación de una trama bien armada del carácter de los hombres valientes con el de los sensatos, cuando el arte real los haya reunido por la concordia y el amor en una vida común y haya confeccionado el más magnifico y excelso de todos los tejidos, y, abrazando a los hombres de la ciudad, tanto esclavos como libres, los contenga en esas red"70. Armar una buena trama no depende sólo de entrelazar los distintos ánimos individuales, sino también de dirigir las restantes artes que conciernen a la comunidad: la jurisprudencia, la retórica, la estrategia. Pero además,

interpretaciones, Castoriadis postula que el mito no se introdujo para desterrar al pastor como metáfora del político sino que el pastor fue el puntapié para presentar el mito, que tenía por objetivo desechar la supuesta autoconstitución de la especie humana postulada por Demócrito. Cornelius Castoriadis, Sobre el político de Platón, pp. 123-126.

${ }^{65}$ La descripción de la era de Cronos se reitera en Leyes, donde se la describe como un momento de felicidad del hombre, en el "que lo poseía todo en abundancia y de manera espontánea." Platón, Leyes, Op. cit., $713 \mathrm{c}$.

${ }^{66}$ Platón, Político, Op. cit., 271e.

${ }^{67}$ Como resume Armando Porati, "la definición del rey como pastor es adecuada en realidad a esta situación." Armando Porati, Teoría política y práctica política en Platón [en Atilio Boron, Comp., La filosofia política clásica. De la Antigüedad al Renacimiento, Buenos Aires, Clacso-Eudeba, 2000] p. 87.

${ }_{68}^{68}$ Michel Foucault, Sécurité, territoire, population, Op. cit., p. 148.

${ }^{69}$ Platón, Político, Op. cit., 275b-c.

${ }^{70}$ Ibídem, 311b-c. 
la metáfora del tejedor vincula la política con el arte de la medida y con la preocupación por el exceso y el defecto, tratando de situar lo conveniente en el justo medio de los extremos ${ }^{71}$.

Político reposiciona la importancia de la ley, y es así que a diferencia de lo que se lee en el libro VIII de República, la tipología de las formas de gobierno toma como variable de distinción el grado de respeto de la ley ${ }^{72}$, criterio que será mantenido en Leyes y será parcialmente retomado por Aristóteles ${ }^{73}$. Dentro de los órdenes que se ajustan a la ley, Platón ordena jerárquicamente la monarquía, aristocracia y democracia (sujeta a la ley), y los que no se ajustan los ordena en democracia (no sujeta a la ley), oligarquía y tiranía. Por encima de estas seis formas de gobierno ubica aquél dirigido por un gobernante dotado de episteme y por ello situado por encima de la ley. Entonces, en boca del Extranjero se lee "así como el piloto, procurando siempre el provecho de la nave y los navegantes, sin establecer normas escritas, sino haciendo de su arte ley, preserva la vida de quienes con él navegan, así también, del mismo modo, ¿de quienes tienen la capacidad de ejercer de esta manera el gobierno, podría proceder el recto régimen político, ya que ellos ofrecen la fuerza de su arte, que es superior a la de las leyes?"74. Es por ello que "quien posee el saber, que es -recordémoslo- quien es realmente un político, hará en su acción personal una cantidad de cosas en virtud de su arte, sin preocuparse para nada de las normas escritas, cuando le parezcan mejores otras reglas frente a las que él ha redactado"75. La aprobación de las tres formas de gobierno que se ajustan a la ley, y la preeminencia del político dotado de episteme, representan dos puntos de vista que oscilan, se solapan y hasta se contradicen en sus reflexiones: "o bien se sitúa en primer plano al verdadero político que, en posesión de la filosofía y superior a sus súbditos y a las leyes, reina otorgando la felicidad al Estado, o bien el autor parece desesperar de la existencia de este hombre superior, y entonces sobresale la ley como única solución para los desórdenes y los males de la comunidad"76. Las reflexiones sobre el verdadero político llevan a Castoriadis a postular que aunque se conceptualice al político como pastor y como tejedor, "la definición adecuada está escondida en el diálogo"77. El político no es el pastor ni el tejedor sino "el epistémon, el que sabe, y el que

${ }^{71}$ Lucas Soares, Platón y la política, Op. cit., pp. 194-196.

72 Platón, Político, Op. cit., 301a y ss.

73 Aristóteles, Política. Buenos Aires, Alianza, 1995, 1289b-1297b.

${ }^{74}$ Platón, Político, Op. cit., 296e-297a. El intelecto como clave para la salvación de la nave y la tripulación es reiterada en el último capítulo de Leyes. Platón, Leyes, Op. cit., 961e.

75 Platón, Político, Op. cit., 300c-d. Una ciudad gobernada por un político "en posesión de semejante 'ciencia' (que no se limitaría a captar las estructuras generales de las situaciones y los seres humanos, sino que alcanzaría a los individuos) sería mucho más feliz que una ciudad gobernada por la ley: pues ésta, por el hecho mismo de ser general, no se aplica jamás de manera perfecta a los casos individuales". Alexandre Koyré, Introducción a la lectura de Platón, Madrid, Alianza, 1996, p. 172.

${ }^{76}$ Giorgio Colli, Platón político, Op. cit., p. 142.

77 Cornelius Castoriadis, Sobre el politico de Platón, Op. cit., p. 43. 
sabe lo que cada uno debe hacer porque posee el verdadero saber" ${ }^{78}$. El poder del epistémon, de este hombre ciencia, está "justificado por el saber político, no tiene ninguna otra limitación que la resultante de su saber mismo"79.

Más allá de la ambivalencia entre un gobierno a través de la ley y un gobernante dotado de episteme, nuevamente situados en la lectura que propone Foucault hay que subrayar que el pastor es evocado pero no es utilizado como una metáfora que explique lo que es propio del ejercicio del poder político, sino más bien, se expone su inutilidad como metáfora explicativa: "se tiene aquí, en este texto, la recusación en buena y debida forma del tema del pastorado."

Incluso con la energía con la que remarca que la metáfora del pastor es insuficiente para dar cuenta de aquello que es propio de la función del magistrado, Foucault no ha logrado sustentar por completo que la metáfora no ha sido utilizada en Grecia para dar cuenta de la función de gobierno. No solamente por ciertas debilidades en el tratamiento de los textos pitagóricos, el olvido de Jenofonte, y las omisiones de República, sino también por ciertos problemas en la presentación de Político. Si bien allí se niega que lo que distingue al magistrado sea asimilable al pastor, no por ello queda descartado que sus funciones -y también las del médico, el pedagogo, etc.- sean pastorales. Si la metáfora del pastor refiere a un gobierno y una crianza sobre los hombres no basta con mostrar, como hace Foucault, que el gobierno de los hombres no es exclusivo del magistrado y puede ser compartido por otros. Es más, el énfasis con el que Foucault subraya que la función pastoral no distingue al magistrado porque la comparte con el médico, el gimnasta y el pedagogo, puede atentar contra sus propios objetivos. Si recordamos que el arte de gobierno de las poblaciones surge como respuesta al Príncipe de Maquiavelo, hay que tener en cuenta que a diferencia de la presentación del florentino, el gobierno de los hombres no lo desarrolla solamente el príncipe -el magistrado- sino que también gobiernan los padres de familia, los pedagogos, etc. ${ }^{81}$ De esta manera, remarcar que el magistrado no puede ser tenido como pastor porque la actividad pastoral también la despliegan otros individuos, lejos de refutar la metáfora del pastor como clave de inteligibilidad del gobierno podría confirmarla: el gobierno pastoral de los hombres la desarrolla el magistrado, pero también el pedagogo y el médico.

78 Ibidem, p. 54.

${ }^{79}$ Cornelius Castoriadis, Sobre el político de Platón, Op. cit., p. 141. Sin embargo, aunque resulta de vital importancia al interior del diálogo, Platón no explica ni desarrolla la conceptualización del político como epistémon sino que esta es una falaz premisa del diálogo. Cornelius Castoriadis, Sobre el político de Platón, Op. cit., p. 74.

${ }^{80}$ Michel Foucault, Sécurité, territoire, population, Op. cit., p. 150.

${ }^{81}$ Ibidem, p. 96. 


\section{Notas finales}

Si en sus estudios sobre el poder en la modernidad había descartado la importancia de los autores más relevantes de la teoría política, en la genealogía del gobierno de los hombres Foucault analiza con cierto detalle la obra de Platón. Al momento de realizar una genealogía sobre el gobierno de los hombres, localiza un antecedente remoto en el poder pastoral y su hipótesis indica que no proviene de Grecia ni de Roma, sino que tiene un antecedente hebreo y un poderoso desarrollo con la Iglesia Católica. En este marco sugirió que la metáfora del pastor como grilla de inteligibilidad del gobierno de los hombres era ajena el pensamiento griego, y se encuentra explícitamente rechazada en la obra de Platón.

Si bien encuentro algunos olvidos y omisiones en los desarrollos de Foucault, puesto que no repara en los trabajos de Jenofonte y la reseña de República resulta insuficiente, en este trabajo ha intentado subrayar algunas inconsistencias en la lectura que propone de Político. Según entiendo, sus desarrollos no solamente no logran sostener que la metáfora del pastor sea ajena el pensamiento griego, sino que su presentación parece cuadrar perfectamente con la conceptualización que él mismo propone del gobierno de los hombres. Foucault ha enfatizado que no puede caracterizarse al político como pastor porque la tarea del cuidado de los hombres también la desarrollan el médico, el gimnasta y el pedagogo, pero paradójicamente, con esta afirmación parece mostrar aquello que es propio de las artes de gobierno de los hombres tal como se despliegan a partir de la modernidad: que la tarea del gobierno no es privativa del político, el magistrado o el príncipe. 


\section{Referencias bibliográficas:}

Alesio, David, La biopolitica foucaulteana: desde el discurso de la guerra hacia la grilla de la gubernamentalidad, "A Parte Rei. Revista de Filosofía, 60 (2008).

Aristóteles, Metafisica, Madrid, Gredos, 1970.

Política. Buenos Aires, Alianza, 1995.

Badiou, Alain, La República de Platón, Buenos Aires, Fondo de Cultura Económica, 2013.

Balaudé, Jean-François, Le triptyque Republique, Politique, Lois: perspectives [en Jean-François Balaudé, Ed., D'une cite possible. Sur le Lois de Plato, Nanterre, Presses de Nanterre, 1995].

Castoriadis, Cornelius, Sobre el político de Platón, Madrid, Trotta, 2004.

Castro-Gómez, Santiago, Historia de la gubernamentalidad., Razón de Estado, liberalismo y neoliberalismo en Michel Foucault, Bogotá, Siglo del Hombre Editores, 2010.

Chignola, Sandro, Foucaulty la política de los gobernados. Gubernamentalidad, formas de vida, subjetivación, "Deus Mortalis", 9 (2010).

Colli, Giorgio, Platón político, Madrid, Siruela, 2008.

Deleuze, Gilles, Guattari, Félix, Qu' est-ce que la philosophie?, Paris, Les éditions de minuit, 1991.

Foucault, Michel, L'ordre du discours, Paris, Gallimard, 1971.

The Subject and Power [en Hubert Dreyfus, y Paul Rabinow, Michel Foucault. Beyond structuralism and hermeneutics, Chicago, Chicago University Press, 1983].

Histoire de la sexualité 2. L'usage des plaisirs, Paris, Gallimard, 1984.

L'herméneutique du sujet. Cours au Collège de France (1981-1982), Paris, Gallimard- Seuil, 2001.

De l'archéologie à la dynastique [en Dits et écrits I, Paris, Gallimard, 2001, $\left.\mathrm{n}^{\mathrm{o}} 119\right]$.

La vérité et les formes juridiques [en Dits et écrits I, Paris, Gallimard, 2001, $\left.\mathrm{n}^{\mathrm{o}} 139\right]$.

La philosophie analytique de la politique [en Dits et écrits II, Paris, Gallimard, 2001, nº 232].

Sexualité et pouvoir [en Dits et écrits II, Paris, Gallimard, 2001, $\mathrm{n}^{\circ}$ 233].

«Omnes et singulatim»: vers une critique de la raison politique [en Dits et écrits II, Paris, Gallimard, 2001, $\left.\mathrm{n}^{\mathrm{o}} 291\right]$.

Usage des plaisirs et techniques de soi [en Dits et écrits II, Paris, Gallimard, 2001, $\left.\mathrm{n}^{\circ} 338\right]$.

À propos de la généalogie de l'éthique: un aperçu du travail en cours [en Dits et écrits II, Paris, Gallimard, 2001, $\mathrm{n}^{\circ}$ 344]. 
La technologie politique des individus [en Dits et écrits II, Paris, Gallimard, 2001, n³64].

Les confessions de Michel Foucault (Entretien avec R.P. Droit) [en Le Point, 01/07/2004. Disponible en http://www.lepoint.fr/ actualites-chroniques/2004-07-01/1es-confessions-de-michelfoucault/989/0/116571].

Sécurité, territoire, population. Cours au Collège de France (1977-1978), Paris, Gallimard-Seuil, 2004.

Le gouvernement de soi et des autres. Cours au Collège de France (19821983), Paris, Gallimard-Seuil, 2008.

Le courage de la vérité. Cours au Collège de France (1983-1984), Paris, Gallimard-Seuil, 2009.

Du gouvernement des vivants. Cours au Collège de France (1979-1980), Paris, Gallimard- Seuil, 2012.

Subjectivité et vérité. Cours au Collège de France (1980-1981), Paris, Gallimard- Seuil, 2014.

Gordon, Colin, Governmental Rationality: An Introduction [en Graham Burchell, Collin Gordon, Peter Miller, Eds.: The Foucault Effect. Studies in Governmentality, Chicago, Chicago University Press, 1991].

Foucault in Britain [en Andrew Barry, Thomas Osborne, Nikolas Rose, eds.:

Foucault and political reason. Liberalism, neo-liberalism and rationalities of government, Chicago, Chicago University Press, 1996].

Gros, Frédéric, Michel Foucault, Paris, Presses Universitaires de France, 1996. Grube, George, El pensamiento de Platón, Madrid, Gredos, 1994.

Hindess, Barry, Discourses of power: From Hobbes to Foucault, Oxford, Blackwell, 1996.

Homero, Odisea, Madrid, Gredos, 1993.

Ilíada, Madrid, Gredos, 1996.

Jenofonte, Ciropedia. Madrid, Gredos, 1987.

Recuerdos de Sócrates. Memorabilia, Madrid, Gredos, 1993.

Koyré, Alexandre, Introducción a la lectura de Platón, Madrid, Alianza, 1996. Lemke, Thomas, Foucault, Governmentality, and Critique, "Rethinking Marxism: A Journal of Economics, Culture \& Society,” 14, 3 (2002).

Lisi, Francisco, Introducción [en Platón, Diálogos VIII. Leyes, Madrid, Gredos, 1999].

O'Malley, Pat, Experimentos en gobierno. Analíticas gubernamentales y conocimiento estratégico del riesgo, "Revista argentina de sociología", 8 (2007).

Pabón, Juan Manuel, Fernández-Galiano, Manuel, Introducción [en Platón, Leyes, Centro de Estudios Políticos y Constitucionales, Madrid, 1999]. Platón, República. Diálogos IV, Madrid, Gredos, 1988. 
Político [en Diálogos V, Madrid, Gredos, 1988].

Critias [en Diálogos VI, Madrid, Gredos, 1992].

Leyes. Diálogos IX, Madrid, Gredos, 1999.

Porati, Armando, Teoría política y práctica política en Platón [en Atilio Boron, Comp., La filosofía política clásica. De la Antigüedad al Renacimiento, Buenos Aires, Clacso-Eudeba, 2000].

Rose, Nikolas, Identity, Genealogy, History [en Stuart Hall y Paul Du Guy, eds.: Questions of Cultural Identity, London, Sage Publications, 1996].

Powers of Freedom. Reframing political thought, Cambridge, Cambridge University Press, 1999.

Rose, Nikolas, O’Malley, Pat, Valverde, Mariana Governmentality, "Annual Review of Law and Social Science", 2 (2006).

Santa Cruz, María Isabel, Introducción [En Platón, Diálogos $V$, Madrid, Gredos, 1988].

Soares, Lucas. Platón y la política, Madrid, Tecnos, 2010.

Strauss, Leo, The City and Man, Chicago, Chicago University Press, 1964. 